\title{
Microstructural analysis and reliability of monolithic zirconia after simulated adjustment protocols
}

\author{
Eliseo Pablo Chun ${ }^{a}$, Lilian Costa Anami ${ }^{a}$, Estevam Augusto Bonfante ${ }^{b}$, \\ Marco Antonio Bottino ${ }^{a, *}$ \\ a Department of Dental Materials and Prosthodontics, São Paulo State University (Unesp), Institute of Science and \\ Technology, Av. Eng. Fco. José Longo, 777, São José dos Campos, SP, 12245-000, Brazil \\ b Department of Prosthodontics and Periodontology, University of Sao Paulo, Bauru School of Dentistry, Alameda Dr. \\ Octávio Pinheiro Brisolla, 9-75, Bauru, SP, 17012-901, Brazil
}

\section{A R T I C L E I N F O}

Article history:

Received 5 January 2017

Received in revised form

28 March 2017

Accepted 28 April 2017

Keywords:

Zirconium

Prosthesis fitting

Mechanical stress

\begin{abstract}
A B S T R A C T
Objective. To investigate the microstructural properties and reliability of zirconia partially stabilized by yttrium oxide (Y-TZP) for use in monolithic form (Vita YZ HT, Vita Zahnfabrik, Germany) after different clinical adjustment protocols simulation. One-hundred and sixty two Y-TZP discs of high translucency were divided into 6 groups $(n=27)$, according to simulated adjustments: S-Y-TZP as sintered; G-glazed Y-TZP; DbG-Y-TZP with diamond bur adjustment simulation followed by glaze application; GDb-glazed Y-TZP adjusted with diamond bur; DbDrG-Y-TZP with adjustment simulation with diamond bur and polishing kit (diamond rubber), followed by glaze; GDbDr-glazed Y-TZP with adjustment simulation with diamond bur followed by polishing kit.

Methods. After monotonic testing under biaxial flexure $(n=3)$, remaining specimens were distributed into 3:2:3 ratio and tested under step-stress accelerated life testing. Specimens were evaluated in a stereomicroscope and SEM. Microstructure was analyzed by X-ray diffraction and mean surface roughness (Ra). Survival data were used to calculate Weibull's beta value $(\beta)$ and reliability for missions of 300,000 and 600,000 cycles at $200 \mathrm{~N}$. Ra data were statistically analyzed by 1-way ANOVA and Tukey's test (both, $a=5 \%$ ).

Results. The untreated HT zirconia (S) presented a lower probability of survival after 600,000 cycles at $200 \mathrm{~N}$, compared to HT zirconia adjusted with diamond bur and glazed (DbG), and after final polishing with polishing kit (GDbDr). Final polishing with diamond rubber (GDbDr) resulted in a more homogeneous surface compared to glazed samples ( $G$, DbG and DbDrG). Significance. HT zirconia is more likely to present increased survival when glazed after diamond adjustments $(\mathrm{DbG})$ or when it is finished with polishing kit (GDbDr).
\end{abstract}

(c) 2017 The Academy of Dental Materials. Published by Elsevier Ltd. All rights reserved.

\footnotetext{
* Corresponding author. Fax: +55 1239479000.

E-mail addresses: eliseo_87@hotmail.com (E.P. Chun), lianami@gmail.com (L.C. Anami), estevamab@gmail.com (E.A. Bonfante), bottinomarcoantonio@gmail.com (M.A. Bottino).

http://dx.doi.org/10.1016/j.dental.2017.04.024

0109-5641/@ 2017 The Academy of Dental Materials. Published by Elsevier Ltd. All rights reserved.
} 


\section{Introduction}

Minimal occlusal thickness and tooth preservation lead to the necessity of using restorations thinner than conventionally indicated. In posterior regions where masticatory loads are higher, polycrystalline ceramics may be the first option due to their transformation toughening properties that are not present in other all-ceramic materials [1].

Zirconia is a polymorphic material presenting three phases, according to temperature: monoclinic (from room temperature to $1700^{\circ} \mathrm{C}$ ), tetragonal (between $1700^{\circ} \mathrm{C}$ and $2370^{\circ} \mathrm{C}$ ), and cubic (above $2370^{\circ} \mathrm{C}$ until its melting). To be able to maintain the zirconia in the tetragonal phase at room temperature, stabilizing oxides are used, such as yttrium oxide. A tetragonal zirconia partially stabilized by yttrium (Y-TZP) offers high mechanical strength due to its ability to resist crack propagation (fracture toughness) [2,3].

Y-TZP may be engineered as a high translucent ceramic (Y-TZP HT) to be fabricated by computer-aided design and computer-aided manufacturing (CAD/CAM), with indications for monolithic use. During prostheses installation, occlusal, proximal and cementation surface adjustments are often required by means of diamond rubbers with different grits, as per manufacturers instructions.

Clinical adjustment of the ceramic surface can lead to crack initiation tending to propagate toward surfaces under tensile stress. The damage can also be accompanied by phase transformation. Appropriate polishing with diamond rubbers can reduce the surface roughness of the Y-TZP, though roughness seems to not influence the phase change in the material [4]. However, the surface roughness plays an important role in the relationship with the opposing tooth, since polished and glazed ceramics have better mechanical behavior [5].

Whereas single load to failure testing lead to abrupt and catastrophic fractures of dental ceramics, failure usually occurs by accumulated fatigue after prolonged low load cycling [6]. There are limited data in the literature on the probability of survival of Y-TZP HT ceramics after clinical adjustment procedures. The purpose of this in vitro study was to evaluate the effect of different occlusal adjustment techniques on monolithic zirconia, before or after the glaze layer application, on the reliability and microstructural characteristics.

\section{Materials and methods}

\subsection{Specimen preparation}

One hundred and sixty two discs were prepared. A ring device was glued onto the top surfaces of the CAD/CAM blocks (Vita YZ HT, Vita Zahnfabrik, Germany) to round them until $14 \mathrm{~mm}$ diameter cylinders were obtained. The cylinders were then cut into several disks in a cutting machine (ISOMET 1000, Buehler, Lake Bluff, USA). Specimens attained final dimensions of $12 \mathrm{~mm}$ in diameter [7] and $0,8 \mathrm{~mm}$ thick after sintering. Ceramic discs were divided into 6 groups $(n=27)$, according to simulated adjustments as follows: S- Y-TZP as sintered; G- glazed Y-TZP; DbG- Y-TZP with diamond bur adjustment simulation followed by glaze application; GDb- glazed Y-TZP adjusted with diamond bur; DbDrG- Y-TZP with adjustment simulation with diamond bur and polishing kit (diamond rubber), followed by glaze; GDbDr- glazed Y-TZP with adjustment simulation with diamond bur followed by polishing kit.

For glazed groups, the Plus Glaze Body Spray (Vita Zahnfabrik, Germany) was applied on a $12 \mathrm{~mm}$ diameter surface.

\subsection{Grinding and polishing on the surface}

To simulate the clinical adjustment procedure with diamond burs, a 120-grit resin-bonded diamond disk, which corresponds to a $151 \mu \mathrm{m}$ diamond bur (blue) [8], was used to polish the $12 \mathrm{~mm}$ diameter surface at a $500 \mathrm{rpm}$ speed for $20 \mathrm{~s}$ mounted in a polishing machine (Automet/Ecomet 250, Buehler, Lake Bluff, USA). For diamond rubber polishing, the Step 1 Pre-polishing Kit (Suprinity Polishing Set, Vita Zahnfabrik, Germany) was used at 7000-12,000 rpm speed for $15 \mathrm{~s}$, followed by Step 2 High Brightness with 4000-8000 rpm for $15 \mathrm{~s}$, both mounted on a laboratory motor handpiece (MF Perfecta 9975, W\&H, Laufen, Germany).

\subsection{Surface roughness}

Six specimens of each group were evaluated for surface roughness obtained by the simulations with a contact rugosimeter (Mitutoyo Corporation, Tokyo, Japan). Three parallel readings per sample were performed, and the Ra parameter was evaluated. A representative sample of each group was also evaluated in digital optical profilometer (Wyko NT model 1100, Veeco, Tucson, USA). The information obtained from profilometer was transferred to the computer through the Wyko software Vision 32 (Veeco, Tucson, USA) to generate threedimensional images.

After confirming the normality assumptions, the roughness data ( $\mathrm{Ra}$ parameter) were tabulated for descriptive statistics of each group and then submitted to 1-way analysis of variance (ANOVA), followed by means test (Tukey), both with $\alpha=0.05$, using Statistix 8.0 software (Analytical Software, Tallahasse, USA).

\subsection{Mechanical testing and reliability analysis}

Three specimens of each group underwent single load-tofailure (SLF) testing at a cross-head speed of $1 \mathrm{~mm} / \mathrm{min}$ in an universal testing machine (EMIC DL 100, Sao Jose dos Pinhais, Brazil), according to ISO 6872:2015 [7], under water. To accelerate specimens' failure, step-stress accelerated life testing (SSALT) was employed using an electrodynamic testing machine. Specimens of all groups were assigned to three stepstress profiles (based on mean SLF values), named based on the load increase in which a specimen is fatigued to reach a certain level of load, following the 3:2:3 ratio and designated as mild $(n=9)$, moderate $(n=6)$, and aggressive $(n=9)$. Twenty-four specimens of each group were cycled in a fatigue testing equipment (Biocycle, Biopdi, Sao Carlos, Brazil) with the samples immersed in distilled water, positioned in a device identical to that used in the monotonic test and with the treated surface facing the tensile side. Considering the $4 \mathrm{~Hz}$ frequency used, each period of $8 \mathrm{~h}$ of cycling corresponded to 115,200 cycles and each period of $12 \mathrm{~h}$ equals 172,800 cycles (Fig. 1). All mechanical tests were performed using a piston- 


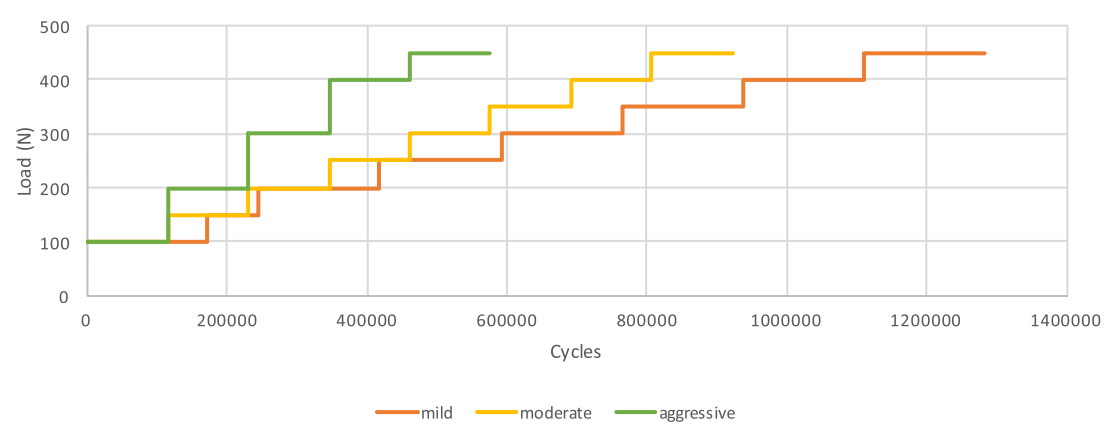

Fig. 1 - The graph represents the step-stress profiles utilized for the accelerated fatigue testing based on the mean value of the single load to failure (SLF). Maximum load was $450 \mathrm{~N}$ and maximum cycles were 1.282,400.

Table 1 - Reliability values, in $\%$, of the experimental groups for missions of 300,000 and 600,000 cycles at $200 \mathrm{~N}$ with upper and lower limit of $90 \%$ confidence interval.

\begin{tabular}{|c|c|c|c|c|c|c|c|}
\hline & & $\mathrm{s}$ & G & DbG & $\mathrm{GDb}$ & DbDrG & GDbDr \\
\hline \multirow[t]{3}{*}{300.000 at $200 \mathrm{~N}$} & $90 \%$ upper & 0,98 & 0,99 & 1,00 & 0,99 & 1,00 & 1,00 \\
\hline & Reliability & 0,96 & 0,97 & 0,99 & 0,98 & 0,98 & 0,99 \\
\hline & $90 \%$ lower & 0,88 & 0,92 & 0,96 & 0,93 & 0,93 & 0,97 \\
\hline \multirow[t]{3}{*}{600.000 at $200 \mathrm{~N}$} & $90 \%$ upper & 0,79 & 0,92 & 0,99 & 0,92 & 0,99 & 1,00 \\
\hline & Reliability & 0,66 & 0,84 & 0,96 & 0,85 & 0,93 & 0,98 \\
\hline & $90 \%$ lower & 0,49 & 0,68 & 0,84 & 0,70 & 0,58 & 0,87 \\
\hline
\end{tabular}

on-three-ball device [7], with a flat punch stainless steel load applicator of $1.4 \mathrm{~mm}$ diameter. Ground surfaces were always positioned on the tensile stresses side.

Based upon the step-stress distribution of failures, use level probability Weibull curves were calculated (Synthesis 9, Alta Pro, ReliaSoft, Tucson, USA) using a power law relationship for damage accumulation. Reliability for a mission of 300,000 cycles and 600,000 at $200 \mathrm{~N}$ (90\% two-sided confidence interval) was calculated for comparison between the groups.

\subsection{Scanning electron microscopy (SEM) /} fractographic analysis

All samples were first inspected in light-polarized stereomicroscopy (Discovery V20 Zeiss, Jena, Germany) and representative samples were then gold sputtered (Emitech SC7620 Sputter Coater, East Sussex, Great Britain) for qualitative fractographic analysis under scanning electron microscopy (SEM Inspect S50, FEI Company, Brno, Czech Republic).

The images obtained by stereomicroscope, optical profilometer and SEM were qualitatively analyzed and described.

\subsection{X-ray diffraction (XRD) phase analysis}

To evaluate potential phase transformation due to simulated adjustments and fatigue, two fractured specimens from each group were evaluated by X-ray difractometry (XRD; Philips X'pert PRO MRD, Almelo, Netherlands). The analysis was carried out in partnership with the LAS/INPE laboratory (Associated Laboratory of Sensors and Materials of the National Institute of Space Research, Sao Jose dos Campos, Brazil). CuK radiation with $\mathrm{Cu}=0.15418 \mathrm{~nm}$ wavelength was used in a range $(-2)$ between $10^{\circ}$ and $100^{\circ}$, scanning speed of $2^{\circ} \mathrm{min}^{-1}$, voltage of $40 \mathrm{kV}$ and current of $20 \mathrm{~mA}$ (Philips PW 1830/1840, Almelo, Netherlands).

The XRD data were evaluated by identifying the crystalline phases after comparing the experimental spectra with standard diffraction spectra of the JCPDS (Joint Committee on Powder Diffraction Standards) and ICSD (Inorganic Crystal Structure). HighScore software (Philips X'pert PANalytical, Almelo, The Netherlands) helped with the attributions of the spectra.

\section{Results}

\subsection{Mechanical testing}

Three specimens did not fail through step-stress fatigue testing, 2 from GDbDr group (mild profile) and 1 from DbDrG group (aggressive profile). Failure load analysis of tested specimens showed the following $\beta$ values: $\mathrm{S}=3.22 ; \mathrm{G}=2.74 ; \mathrm{DbG}=2.09$; $\mathrm{GDb}=2.95 ; \mathrm{DbDrG}=1.79 ; \mathrm{GDbDr}=1.82$.

The step-stress use-level probability Weibull plot is presented in Fig. 2. The reliability values presented in percentages indicated that, the probability of survival for a mission of 300,000 cycles at $200 \mathrm{~N}$ was not significantly different between the experimental groups, since there was an overlap of the confidence intervals (Table 1). For a mission of 300,000 cycles, cumulative damage reaching $200 \mathrm{~N}$, resulted in $1-4 \%$ probability of failure, regardless of the type of simulated adjustment performed on the surface.

The reliability calculated for a mission of 600,000 cycles at $200 \mathrm{~N}$ showed that the accumulated damage would result in $34 \%$ failures of the untreated samples (Table 1). Glazed samples with diamond bur simulation and polishing kit (GDbDr) presented a $2 \%$ probability of failure. Due to the absence of overlapping of the confidence intervals, it was observed that 


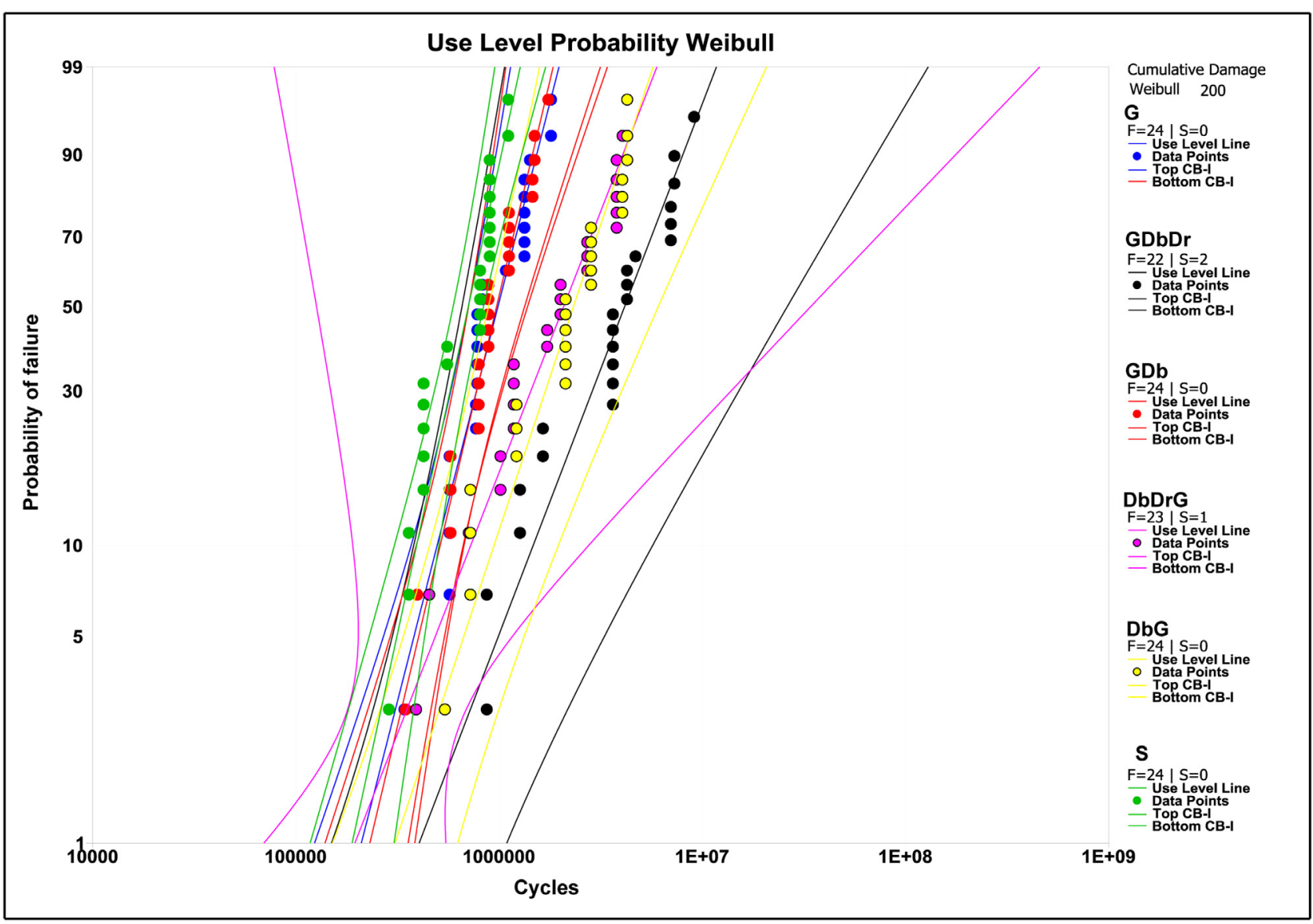

Fig. 2 - Weibull's graph of multiplots of the probability of failure at $200 \mathrm{~N}$ of the experimental groups assuming $90 \%$ confidence interval as function of elapsed cycles.

\begin{tabular}{lc}
$\begin{array}{l}\text { Table 2 - Mean, standard deviation (SD) and } \\
\text { homogeneous Tukey's groups for Ra data (in } \boldsymbol{\mu m} \text { ). }\end{array}$ \\
\hline Groups & $\begin{array}{c}\text { Ra } \\
\text { Mean } \pm \text { SD }\end{array}$ \\
\hline S & $0,72 \pm 0,48^{\mathrm{AB}}$ \\
G & $1,12 \pm 0,18^{\mathrm{AB}}$ \\
DbG & $1,32 \pm 0,33^{\mathrm{B}}$ \\
GDb & $0,61 \pm 0,47^{\mathrm{AB}}$ \\
DbDrG & $1,45 \pm 0,42^{\mathrm{B}}$ \\
GDbDr & $0,21 \pm 0,11^{\mathrm{A}}$ \\
\hline
\end{tabular}

Identical letters indicate absence of statistically significant difference.

samples without glaze or surface treatment (S) presented significantly lower reliability compared to the groups DbG and GDbDr, whereas G, DbG and DbDrG were similar to the other evaluated groups.

\subsection{Fractographic analysis}

The fractographic analysis indicated that the defects likely originating the fractures were mostly on the surface of the ceramic discs (Figs. 3 and 4). Defects were observed due to the simulation procedures of clinical adjustments. Some specimens presented small damages caused by the load applicator on the compression side, but none were associated with the fracture's origin.

\subsection{XRD}

Fig. 5 shows crystalline phase identification graphs obtained by the X-ray diffraction analysis for the samples of each experimental group. It is observed that there was no significant change in the zirconia crystallography after the simulations of clinical adjustment. The ZrT demarcated peaks show the presence of tetragonal phase, indicating that the faults occurred due to the alteration of the microstructure of the material and not due to phase transformation.

\subsection{Surface roughness}

Fig. 6 presents the images obtained in 2D and 3D digital optical profilometry for each of the groups. The surface of S presented deeper polishing grooves. On the surface of the specimens of groups G and DbDrG glaze accumulation "islands" can be observed. These specimens were visually more irregular compared to the other groups. This fact was not observed in the representative image of the DbG group. GDb presented more pronounced peaks and valleys than GDbDr.

The micrographs of the surfaces depicted grooves resulting from polishing in the sintered sample (S, Fig. 6), shown to 


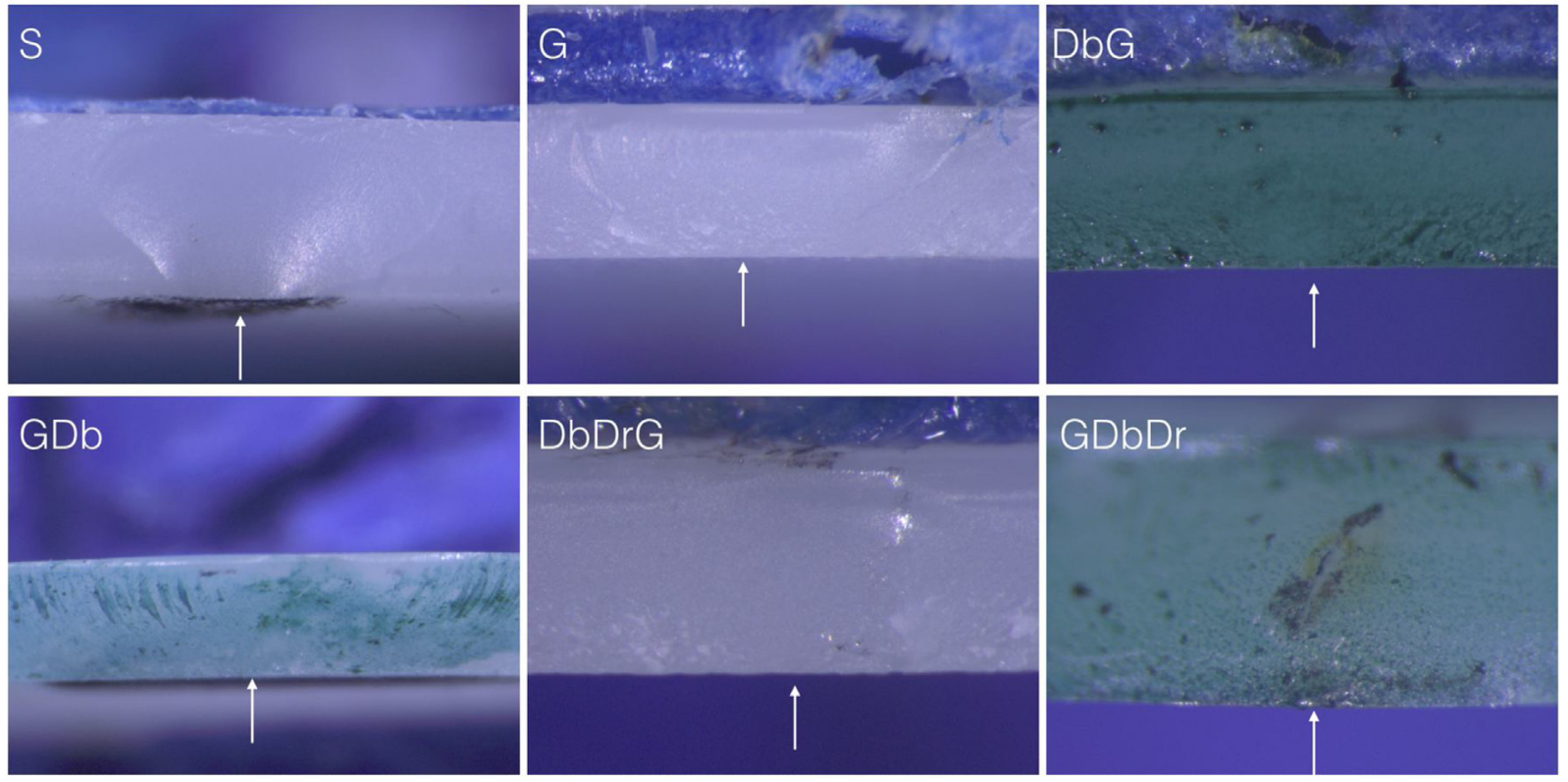

Fig. 3 - Representative optical images of partial failure of the zirconia. The white arrows indicate the probable origin of the fracture on the tensile side.
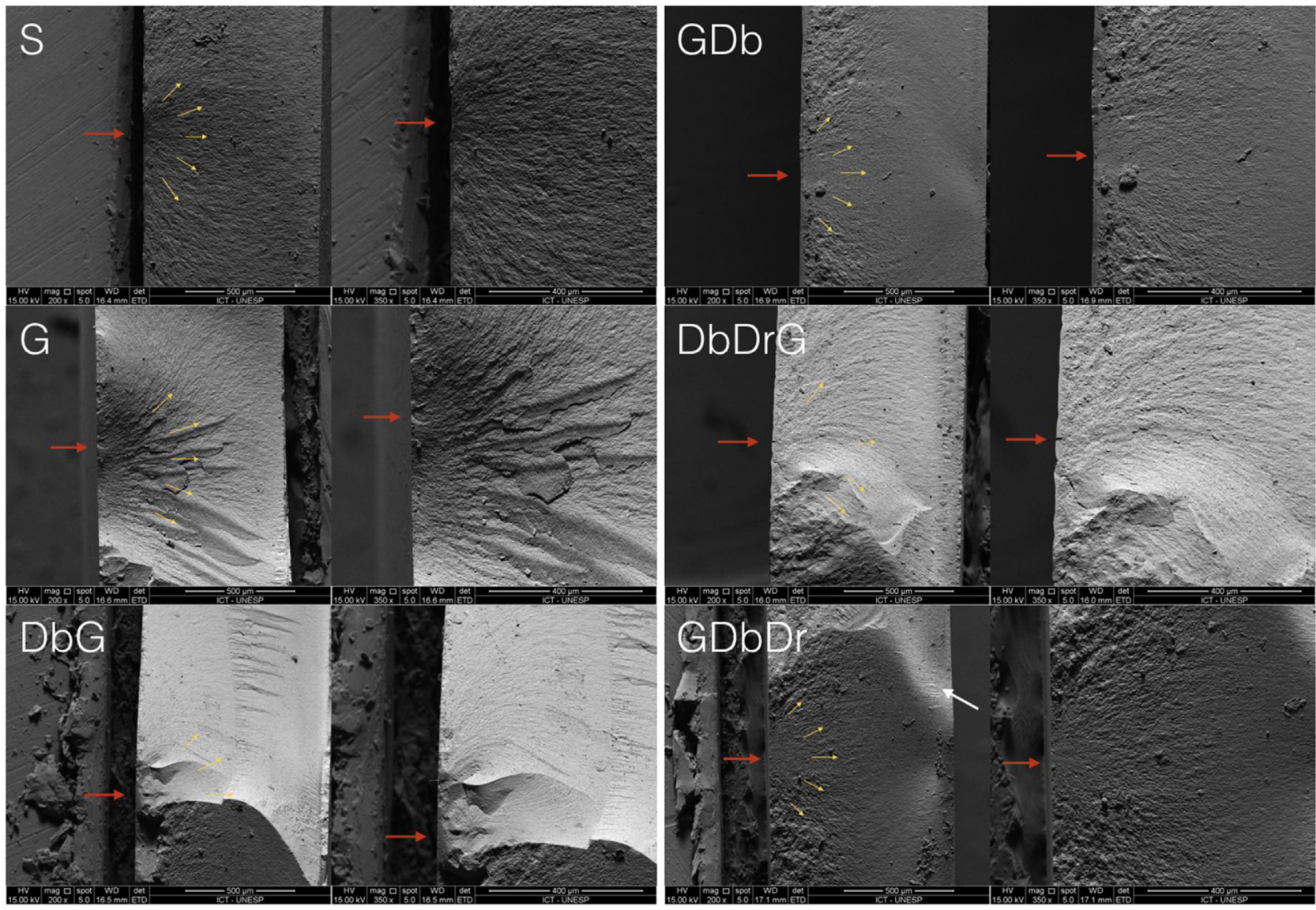

Fig. 4 - Representative SEM images of treated zirconia surfaces of tested groups. The yellow arrows indicate the propagation of the fracture, the red arrows indicate the probable origin of the fractures and the white arrow indicates the compression curl. (For interpretation of the references to color in this figure legend, the reader is referred to the web version of this article.) 

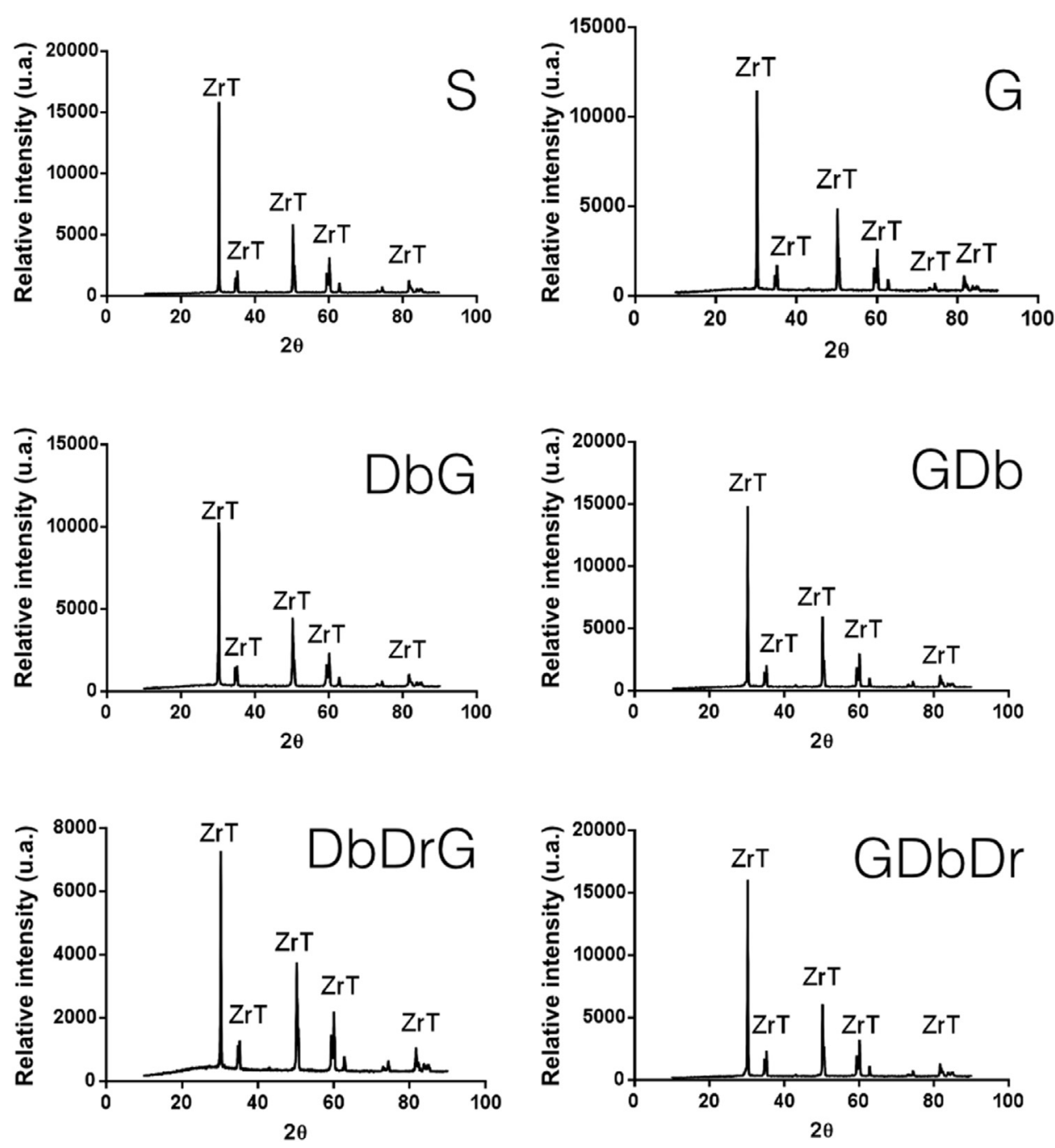

Fig. 5 - Identification graphs of the crystalline phases after comparison of the experimental spectra with diffraction spectra.

be more irregular than the groups that received glaze ( $G$ and DbDrG, Fig. 6). It is observed that the glaze was not able to cover the surface of the zirconia previously adjusted with diamond bur (DbG; Fig. 6), thus appearing as "islands" (Fig. 6).

The 1-way ANOVA detected a statistically significant difference between the roughness resulting from the simulated adjustments for the Ra parameter $(p=0.010)$. The application of glaze followed by grinding with diamond bur and polishing kit (GDbDr) resulted in the lowest values of Ra, similar to the roughness of Y-TZP HT as sintered (S) or glazed (G), and glazed and diamond bur adjustment simulation (GDb) (Table 2).

\section{Discussion}

The step-stress fatigue test used in this study is a relatively new method in dental research and has not yet been used in the evaluation of high translucent zirconia. The reason for using different loading profiles is based on the need to distribute the failure in different loads, allowing better fore-casting statistics, narrowing the confidence limits, based on Weibull's calculations. In this study, the samples were mechanically tested under three loading profiles (mild, moderate or aggressive) until the failure of the sample, or the suspension of the sample at the end of the prescribed load/cycle mission. It was observed that the probability of failure of all experimental groups was similar in the simulation of 300,000 cycles at $200 \mathrm{~N}$, with average survival probability between 96 and 99\%. However, in the evaluation of a longer mission $(600,000$ cycles under the same load), the probability of failure increased significantly for the $\mathrm{S}$ group, reaching a survival average of $66 \%$. Thus, the tested hypothesis was partially accepted, since the group that received glaze application and polishing treatment with polishing kit after the fatigue test (GDbDr) presented a probability of survival of a mission of 600,000 cycles at $200 \mathrm{~N}$ higher than the samples without treatment (group S), but similar to the other evaluated groups (Fig. 7).

The strength of monolithic zirconia can be affected by its phase transformation when subjected to stresses. The phase transformation mechanism acts as a barrier that hinders the propagation of the crack, increasing the fracture toughness of zirconia [9]. Phase transformations may occur due to low temperature degradation and/or mechanical stress, occurring at 

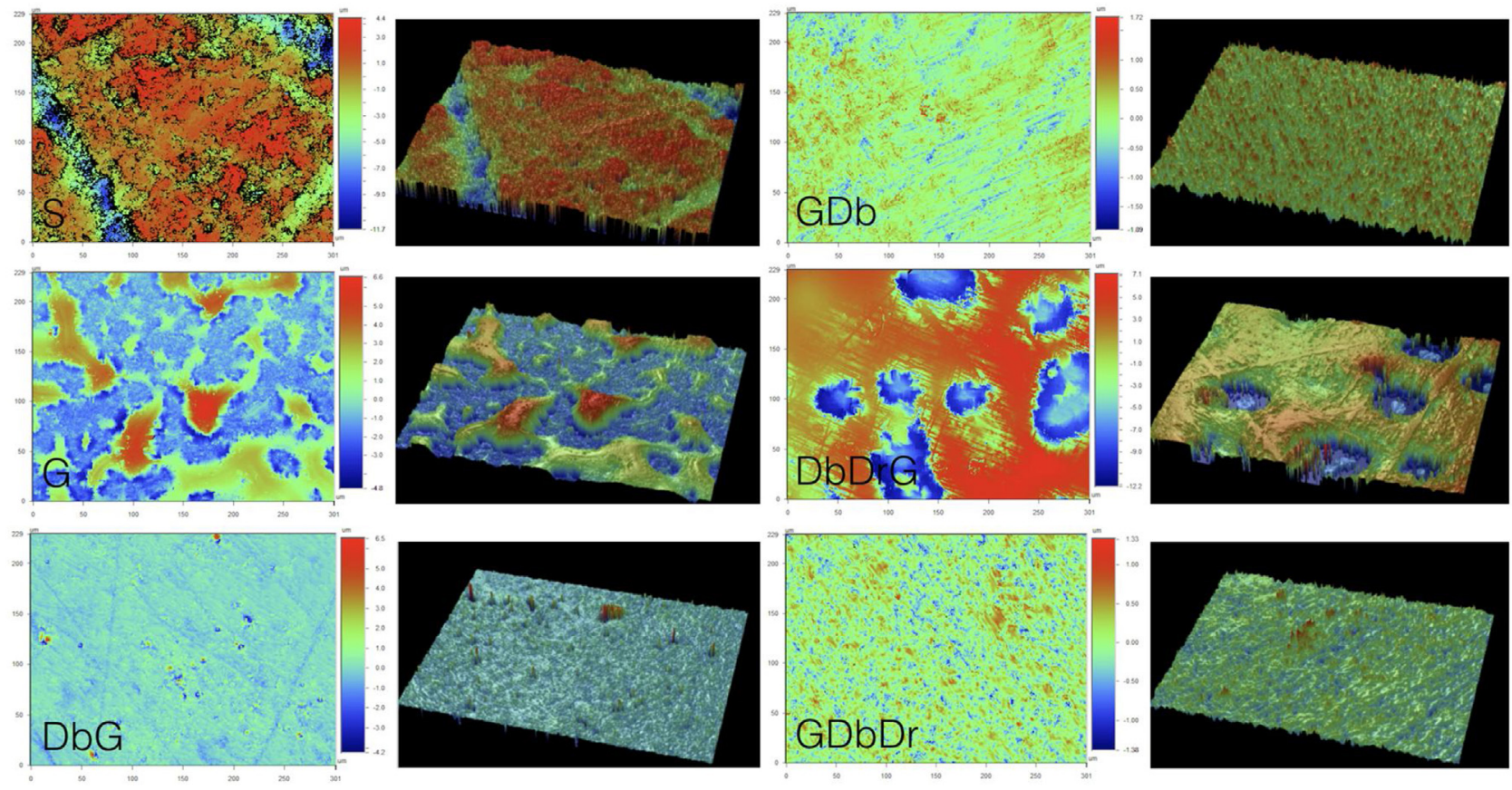

Fig. 6 - Representative digital perfilometer images of treated zirconia surfaces of tested groups.

the surface. In this study, no significant changes were observed in zirconia crystallography after the simulations of clinical adjustment, corroborating with the conclusions of Kosmac et al. $[10,11]$, who reported that wear with diamond burs did not promote an increase in monoclinic phase. Our results demonstrated that resin bonded diamond disks used to simulate higher standardization of diamond-bur wear did not result in phase changes evaluated by X-ray diffraction, as reported by Huh et al. [12].

In this study, roughness resulting from each simulated occlusal adjustment protocol was also compared. As expected, the roughness values of the polished samples were lower than the others. The final polishing with polishing kit generated the smooth surface (GDbDr). This supports the hypothesis that polishing is able to remove some of the defects induced by the adjustments and, although it also generates defects, they have a more favorable distribution reducing the surface roughness pattern, as reported by Preis et al. [4]. Ceramics with high roughness patterns lead to natural enamel wear, although zirconia does not become as aggressive to the antagonist as roughened feldspathic porcelain $[13,14]$.

The micrographs of the groups where surfaces were finished with glaze application (G, DbG and DbDrG) showed glaze accumulation "islands", which were also verified in G and DbDrG groups thus corroborating with the results of average roughness. The DbG profilometry (Fig. 6) did not show such "islands" accumulation probably because of the limited evaluation area allowed by the equipment. Both, the profile image and the micrograph of the GDbDr presented fairly regular surfaces, an observation that can be correlated with the roughness results shown to be lower compared to the samples that received the glaze layer as the final processing. The reduction of roughness after polishing, observed herein, was also observed by Goo et al. [15]. Although there have been statisti- cal and visual differences of roughness between the simulated adjustment protocols of the samples, surface defects did not seem to be related with the origin of the fractures.

It is known that monolithic zirconia crowns exhibit greater fracture resistance than bilayered ones [16] and that the thickness of full contour ceramic restorations influences fractures resistance [17]. According to the ISO 6872:2015 [7], specimens for the biaxial flexural strength test should have a diameter of $12 \mathrm{~mm}$ and a thickness of $1.2 \mathrm{~mm}$, but the literature presents several papers that used Y-TZP specimens or other ceramics in dimensions other than those required by ISO for biaxial flexural tests [18-26]. Due to a limitation of the fatigue equipment in load application levels, this work used $0.8 \mathrm{~mm}$ thick samples after monotonically testing samples of varying thicknesses of 0.6 to $1.4 \mathrm{~mm}$ in a pilot study. Despite the expected difference between the maximum load fracture values, the calculated strength remained relatively constant across the thicknesses. It was also realized that thicknesses of $1 \mathrm{~mm}$ and above led to suspension of all samples, even when fatigued over one million cycles at loads exceeding $400 \mathrm{~N}$. Therefore, whereas $0.8 \mathrm{~mm}$ thickness is less than the recommended by the norm, it is a clinically relevant thickness when indicating monolithic zirconia crowns in posterior areas. According to Nakamura et al. [27] and Sorrentino et al. [28], monolithic zirconia crowns can be made in areas with a minimum occlusal thickness of $0.5 \mathrm{~mm}$, allowing less invasive preparations that may save substantial amount of dental tissue [29].

The comparison between results of in vitro simulation studies of clinical adjustment is hampered by the fact that these procedures are not subject to standardized conditions with variations of time, pressure and load orientation during wear. Pereira et al. [30] made markings with a permanent marker on the surface of the samples, which were worn until the ink mark was extinguished. Hmaidouch et al. [31] stan- 

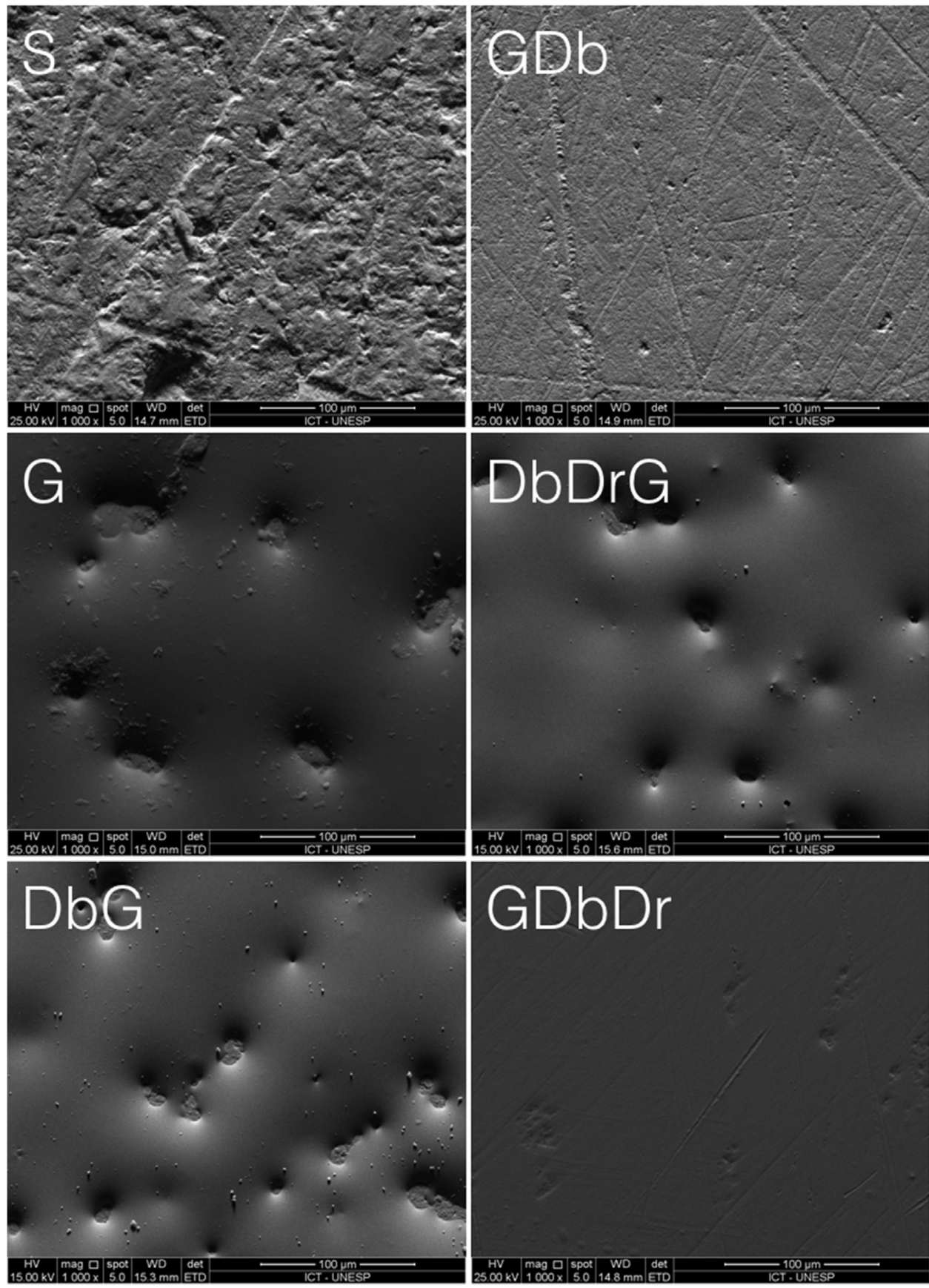

Fig. 7 - Representative SEM images of treated zirconia surfaces of tested groups.

dardized wear parameters $(200,000 \mathrm{rpm}, 15 \mathrm{~s}, 2 \mathrm{~N}$ pressure, 20, 10 and $7.5 \mu \mathrm{m}$ burs) and replaced the diamond burs and rubbers between each sample. Preis et al. [4] used other parameters $(160,000 \mathrm{rpm}, 10 \mathrm{~s}, 1 \mathrm{~N}$, burs from 27 to $76 \mu \mathrm{m})$ and did not replace the grinding instruments between samples. Husain and Özcan [32] used a custom device to position the handpiece parallel to the sample. The present study used polystyrene sanders with speed and grinding time controlled. The granulation of the resin bonded diamond disk 120, for example, corresponds to the granulation of diamond bur of $151 \mu \mathrm{m}$ [8]. Future studies evaluating the standardization conditions that best simulate clinical settings are warranted.
The clinical fracture of ceramic materials does not usually occur abruptly, due to point overload (monotonic load). Fatigue and slow crack growth after a high number of low load cycling are the most common operating mechanisms eventually leading to fractures [6]. These failures occur depending on clinical variables such as the wet medium, direction and distribution of the applied load, type of cementing agent and the bond between the materials involved in the process [33]. In this study, step-stress accelerated life testing was performed with the samples kept submerged in water and fatigue loaded until fracture (or until reaching the end of the loading profile 
when they were considered as suspensions), with a maximum force of $450 \mathrm{~N}$, which can be reached by patients [34].

Despite the contact damage caused by the opposing tooth or restoration, clinical fractures may also start at the cementation surface (under tensile stress) of the restorations [35,36], as the fractures observed in this study, which began on the tensile side and were related to material surface defects. Thus, this test can be considered as an important tool to study and predict the mechanical behavior of ceramic materials such as zirconia with simulated clinical adjustments and can be applied to other types of specimens, such as crowns or fixed dental prostheses, to predict their probability of survival and simulate clinically relevant failure modes.

\section{Conclusion}

Within the limitations of this study and based on the results, it can be concluded that:

A.) Clinical adjustment simulations did not significantly alter the crystallography of zirconia, but the final polishing with a polishing kit resulted in a more homogeneous surface compared to glazed samples.

B.) Untreated HT zirconia is less likely to survive, after 600,000 cycles at $200 \mathrm{~N}$, compared to HT zirconia ground with diamond bur and glazed and after final polishing with a polishing kit.

\section{Acknowledgement}

This study was funded by grants from FAPESP (2012/190787 and 2014/19357-9), CAPES, and Conselho Nacional de Desenvolvimento Cientí́fico e Tecnoloígico (CNPq grant 309475/2014-7).

\section{REFERE N C E S}

[1] Gracis S, Thompson VP, Ferencz JL, Silva NR, Bonfante EA. A new classification system for all-ceramic and ceramic-like restorative materials. Int J Prosthodont 2015;28(May-June (3)):227-35.

[2] Piconi C, Maccauro G. Zirconia as a ceramic biomaterial. Biomaterials 1999;20(January (1)):1-25.

[3] Zhang Y, Sailer I, Lawn BR. Fatigue of dental ceramics. J Dent 2013;41(December (12)):1135-47.

[4] Preis V, Schmalzabauer M, Bougeard D, Schneider-Feyrer S, Rosentritt M. Surface properties of monolithic zirconia after dental adjustment treatments and in vitro wear simulation. J Dent 2015;43(January (1)):133-9.

[5] Lawson NC, Janyavula S, Syklawer S, Maclaren EA, Burguess JO. Wear of enamel opposing zirconia and lithium dissilicate after adjustment, polishing and glazing. J Dent 2014;42(December (12)):1586-91.

[6] Zhang Y, Lee JJ, Srikanth R, Lawn BR. Edge chipping and flexural resistance of monolithic ceramics. J Dent 2013;29(December (12)):1201-8.

[7] ISO Standard No. 6872. Dentistry - ceramic materials. 4th ed. International Organization for Standardization; 2015. p. 1-28.
[8] Kim JW, Covel NS, Guess PC, Rekow ED, Zhang Y. Concerns of hydrothermal degradation in CAD/CAM zirconia. J Dent Res 2010;89(January (1)):91-5.

[9] Kim HT, Han JS, Yang JH, Lee JB, Kim SH. The effect of low temperature aging on the mechanical property \& phase stability of Y-TZP ceramics. J Adv Prosthodont 2009;1(November (3)):113-7.

[10] Kosmac T, Oblak C, Jevnikar P, Funduk N, Marion L. The effect of surface grinding and sandblasting on flexural strength and reliability of Y-TZP zirconia ceramic. Dent Mater 1999;15(November (6)):426-33.

[11] Kosmac T, Oblak C, Maior L. The effects of dental grinding and sandblasting on aging and fatigue behavior of dental zirconia (Y-TZP) ceramics. J Eur Ceram Soc 2008;28:1085-90.

[12] Huh YH, Park CJ, Cho LR. Evaluation of various polishing systems and the phase transformation of monolithic zirconia. J Prosthet Dent 2016;116(September (3)):440-9.

[13] Amer R, Kurklu D, Kateeb E, Seghi RR. Three-body wear potential of dental yttrium-stabilized zirconia ceramic after grinding, polishing, and glazing treatments. J Prosthet Dent 2014;112(November (5)):1151-5.

[14] Mundhe K, Jain V, Pruthi G, Hah N. Clinical study to evaluate the wear of natural enamel antagonist to zirconia and metal ceramic crowns. J Prosthet Dent 2015;114(September (3)):358-63

[15] Goo CL, Yap A, Tan K, Fawzy AS. Effect of polishing systems on surface roughness and topography of monolithic zirconia. Oper Dent 2016;41(July-August (4)):417-23.

[16] Ramos GF, Monteiro EB, Bottino MA, Zhang Y, Marques de Melo R. Failure probability of three designs of zirconia crowns. Int J Periodontics Restor Dent 2015;35(November-December (6)):843-9.

[17] Sun T, Zhou S, Lai R, Liu R, Ma S, Zhou Z, et al. Load-bearing capacity and the recommended thickness of dental monolithic zirconia single crowns. J Mech Behav Biomed Mater 2014;35(July):93-101.

[18] Anusavice KJ, Shen C, Vermost B, Chow B. Strengthening of porcelain by ion exchange subsequent to thermal tempering. Dent Mater 1992;8(May):149-52.

[19] Cattell MJ, Chadwick TC, Knowles JC, Clarke RL. The development and testing of glaze materials for application to the fit surface of dental ceramic restorations. Dent Mater 2009;25:431-41.

[20] Chen YM, Smales RJ, Yip KHK, Sung WJ. Translucency and biaxial flexural strength of four ceramic core materials. Dent Mater 2010;24:1506-11.

[21] Dikicier S, Ayyildiz S, Ozen J, Sipahi C. Influence of core thickness and artificial aging on the biaxial flexural strength of different all-ceramic materials: an in-vitro study. Dent Mater J 2017, http://dx.doi.org/10.4012/dmj.2016-157.

[22] Guess PC, Zhang Y, Kim JW, Rekow ED, Thompson VP Damage and reliability of Y-TZP after cementation surface treatment. J Dent Res 2010;89(6):592-6.

[23] Hjerppe J, Närhi T, Fröberg K, Vallittu P, Lassila LVJ. Effect of shading the zirconia framework on biaxial strength and surface microhardness. Acta Odontol Scand 2008;66:262-7.

[24] Hjerppe J, Vallittu PK, Fröberg K, Lassila LVJ. Effect of sintering time on biaxial strength of zirconium dioxide. Dent Mater 2009;25:166-71.

[25] Lin WS, Ercoli C, Feng C, Morton D. The effect of core material, veneering porcelain, and fabrication technique on the biaxial flexural strength and Weibull analysis of selected dental ceramics. J Prosthodont 2012;21(5):353-62.

[26] Spyropoulou PE, Kamposiora P, Eliades G, Papavasiliou G, Razzoog ME, Thompson JY, et al. Composition, phase analysis, biaxial flexural strength, and fatigue of unshaded versus shaded Procera zirconia ceramic. J Prosthet Dent 2016;116(2):269-76. 
[27] Nakamura K, Harada A, Kanno T, Inagaki R, Niwano Y, Mileding P, et al. The influence of low-temperature degradation and cyclic loading on the fracture resistance of monolithic zirconia molar crowns. J Mech Behav Biomed Mater 2015;47(July):49-56.

[28] Sorrentino R, Triulzio C, Tricarico MG, Bonadeo G, Gherlone $\mathrm{EF}$, Ferrari $\mathrm{M}$. In vitro analysis of CAD-CAM monolithic zirconia molar crowns with different occlusal thickness. J Mech Behav Biomed Mater 2016;14(April (61)):328-33.

[29] Nordhal N, Vult von Steyern P, Larsson C. Fracture strength of ceramic monolithic crown systems of different thickness. J Oral Sci 2015;57(September (3)):255-61.

[30] Pereira G, Amaral M, Cesar PF, Bottino MC, Kleverlaan CJ, Valandro LF. Effect of low-temperature aging on the mechanical behavior of ground Y-TZP. J Mech Behav Biomed Mater 2015;45(May):183-92.

[31] Hmaidouch R, Muller WD, Lauer HC, Weigl P. Surface roughness of zirconia for full-contour crowns after clinically simulated grinding and polishing. Int J Oral Sci 2014;6(December (4)):241-6.
[32] Husain NA, Özcan M. A study on topographical properties and surface wettability of monolithic zirconia after use of diverse polishing instruments with different surface coatings. J Prosthodont 2016;(July).

[33] Kelly JR, Rungruanganunt P, Hunter B, Vailati F. Development of a clinically validated bulk failure test for ceramic crowns. J Prosthet Dent 2010;104(October (4)):228-38.

[34] Palinks M, Nassar MS, Cecílio FA, Siessere S, Semprini M, Machado de Sousa JP, et al. Age and gender influence on maximal bite force and a masticatory muscles thickness. Arch Oral Biol 2010;55(October (10)):797-802.

[35] Scherrer SS, Quinn JB, Quinn GD, Kelly JR. Failure analysis of ceramic clinical cases using qualitative fractography. Int $\mathrm{J}$ Prosthodont 2006;19(March-April (2)):185-92.

[36] Scherrer SS, Quinn GD, Quinn JB. Fractographic failure analysis of a Procera AllCeram crown using setereo and scanning electron microscopy. Dent Mater 2008;24(August (8)):1107-13. 